\title{
Designing Socially-Aware Persuasive Systems: a Proposed Framework
}

\author{
Geovanna Evelyn Taype Espinoza ${ }^{1}$ and Maria Cecilia Calani Baranauskas ${ }^{2}$ \\ ${ }^{1}$ University of Campinas, UNICAMP, Brazil \\ evelynespinozataype@gmail.com, c.baranauskas@gmail.com
}

\begin{abstract}
Every designer wants their computer systems design to be used by their target audiences. However, the lack of formal support from the persuasive design approaches often leads their projects to fail. In this work we propose a framework with methods, artifacts, tools and steps as a guide to design persuasive technologies, drawing on the socially- aware design perspective. A case study briefly presented illustrates the use of the framework. Results suggest potential benefits of the proposed framework.
\end{abstract}

Keywords: Persuasive System Design, Pervasive Technology, Human Computer Interaction, Information System Design, Persuasive Technology.

\section{Introduction}

There have been several investigations regarding the Persuasive System Design, mostly taking as foundation the works of Fogg [5] and Oinas [6] who propose principles and process to design persuasive systems. Mustaquim and Nyström, in their research focused on sustainability, add some principles taking into account the Cognitive Dissonance Theory [7]. Also Stibe, in his research, uses 7 principles (from those already proposed by Oinas) in a framework to design socially influencer systems. Recent works toward the persuasive design frameworks have addressed the sustainability transformation toward wellbeing [8], the process of developing persuasive mobile systems [9] among others. The comparison among these works was made in a previous work [4], showing the points in common and the differences among these researches; additionally, we suggested new aspects to be considered according to the contemporary demands, e.g. ethics [4].

Is important to note that a considerable amount of the literature shows that the principles proposed by the researchers (that were mentioned above) are used to design information systems with persuasive features. However, we have identified in previous work (carried out by Espinoza and Baranauskas) [4] that there is a lack of focus on the design of ubiquitous and pervasive technologies taking into account a socially aware design and the stakeholders' behavior. Taking this into account, in this work we materialize the lack of these factors in a framework as a guide to persuasive systems design. 
This work aims at contributing to the design of information systems by proposing a framework constituted by artifacts, tools, and steps of a process to guide the design of persuasive systems, taking into account the current innovations in technological scenarios. The framework involves concepts, theory, methods, process and a tool for practical purposes that will be available in the OpenDesign platform [11] [3] for all the community of designers, developers, scientists, professionals, scholars and others who want to design information systems with persuasive or influencer features.

Adopting this type of framework to design persuasive systems is important, not only because the intent of any designer is to persuade users to the designed systems, but because these types of systems could help to transform the human behavior aiming at its well-being. Researchers have shown the necessity to design persuasive systems to influence people to adopt good behaviors such as to eat healthy food, make sports, and others [24][25] We want to contribute by guiding and supporting the design of those systems.

The text is organized as follows: section 2 presents the overall idea of the proposed framework and section 3 illustrates an instantiation of parts of the framework in a case study, and section 4 concludes, pointing out further work.

\section{The Proposed Persuasive Design Framework}

The Framework proposed and illustrated in this work recognizes the role of stakeholders in the information system design process as direct influencers of design decisions; as such it draws on the Socially-aware Design process (SAwD) as a base for practical considerations [13], which is grounded in some Organizational Semiotics artifacts [2] [15]. It naturally draws also upon previous work in the field of persuasive systems design such as Fogg [5], Oinas [6], Mustaquim [7] and Stibe [8].

\subsection{Methodological - Foundation}

This research has foundation on the Socially-aware Design, and Persuasive Design Principles. SAwD is a perspective on information systems design that draws on Organizational Semiotics and Co-design artefacts and methods, enabling a collective work on discussions and understanding of the stakeholders' needs and problems toward a socially aware product design [10], [14]. The Organizational Semiotics facilitates understanding of the organizational structure as formal, informal and technical layers of information, to balance social and technical aspects in the prospective information system, Figure 1 shows the Organizational Semiotics semiotic onion, situating the design process. Co-design is a practice that involves dialog not only of the designers but also of people interested in participating and sharing experiences to build the prospective system. Persuasive design principles take foundations mainly in Fogg and Oinas principles [5] [6]. 


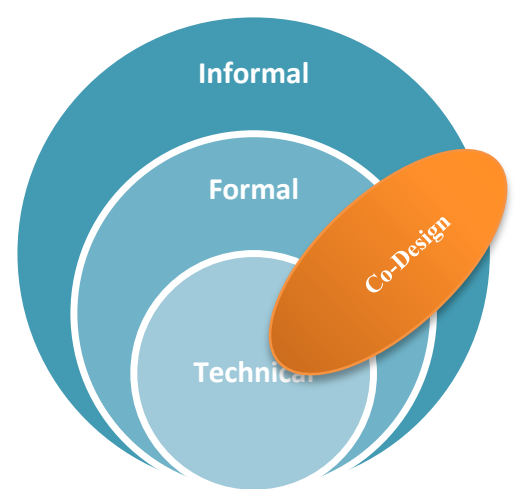

Fig. 1. The Organizational Semiotics Perspective on systems' design [13].

\subsection{A Process to Design Socially-aware Persuasive Systems}

In the proposed approach, the creation of information systems is a process which starts motivated by demands of people in a social context (the outer layer of the semiotic onion) and as such must consider the different stakeholders potentially affected and affecting the system conception. The stakeholders raised by the participants in the codesign of the information system (who are also stakeholders), are then discussed, considering their different relation to the prospective system, represented in different layers (e.g., those who are more directly impacted by the system, those who might indirectly influence the system, those who may legislate over the system, etc.).

The framework proposed is composed by a process with steps as a guide in the design of information systems with persuasive features. The steps to analyze and design persuasive information systems is summarized as shown in Figure 2. The process is composed by six steps; three artifacts are part of the process to guide the design, which support the problem articulation, ideation, requirements discussion and specification they are specifically the Stakeholder Diagram Identification (SDI), the Stakeholder Behavior Identification (SBI) and the Persuasion Frame (PF). 


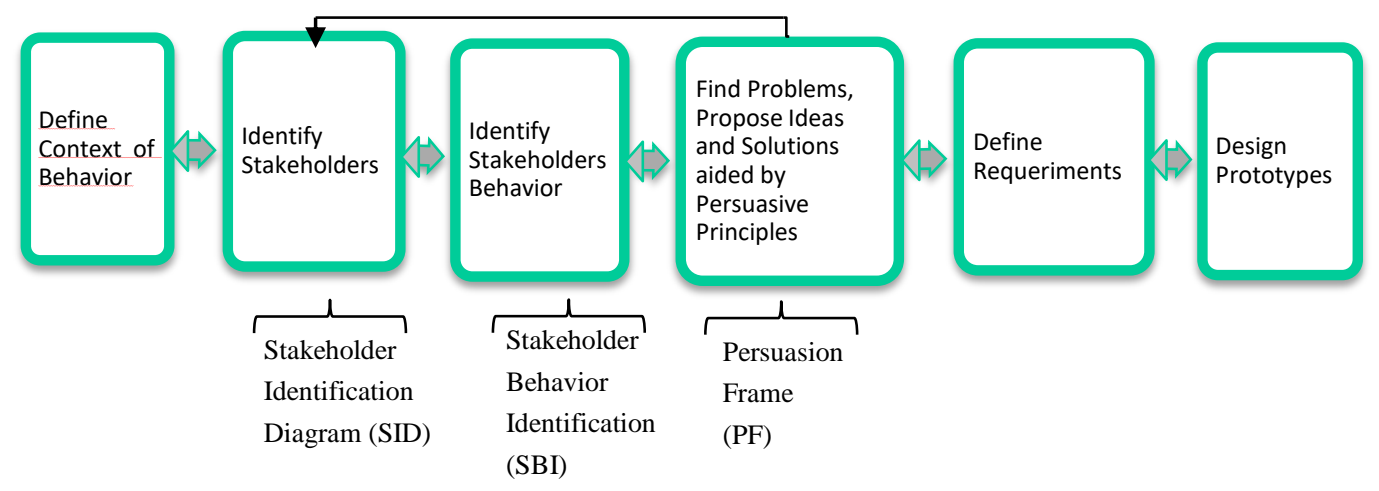

Fig. 2. The process with artifacts to support the socially-aware persuasive system design [4].

The process illustrated in Figure 2 differs from the traditional frameworks because it considers the Co-design of interested parties [13], a collaborative work with the participants (system designers and non-designers) to design systems under the umbrella of the socially-aware design theory [26]. Additionally, it considers the participants' behavior styles (as stakeholders) towards a specific behavior change, and persuasive principles.

A more detailed account of each step is given in the following sections.

\subsubsection{Define Context of Behavior}

The context of persuasion is defined by the problem and the intended target behavior. A first step in the process is defining the problem in behavioral terms, along with the definition of the target behavior (e.g. to consume healthy food) [19] [20].

\subsubsection{Identify Stakeholders}

The stakeholders identification phase supports the identification of stakeholders from different layers of the semiotic onion; from the actors that are directly involved in the persuasive system design to people that might not directly use the system, but can be affected by it, or might legislate on it. The artifact that supports this step is the Stakeholders Identification Diagram SID [2] as shown at Figure 3. (available in URL https://persuasive.opendesign.ml/). 


\section{(ת OPENDESIGN}

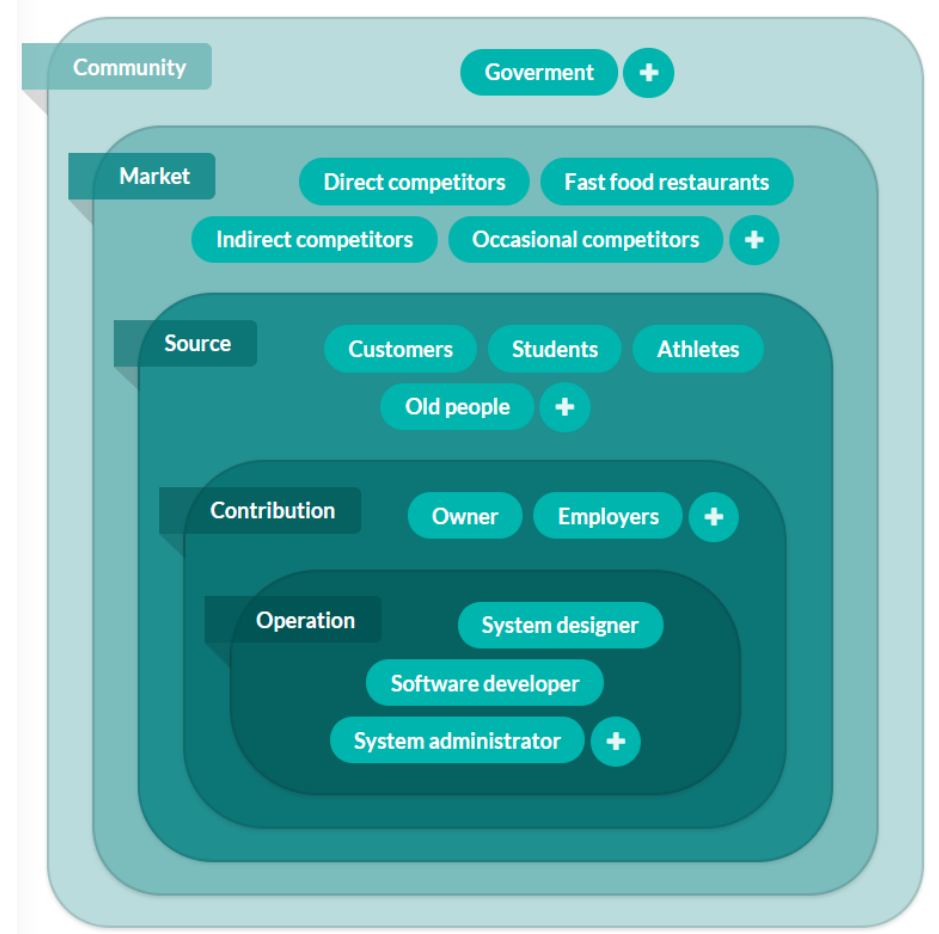

Fig. 3. Stakeholder Diagram Identification [3] [11]. URL

https://persuasive.opendesign.ml/. Data shown at the SID are from the case study.

Figure 3 illustrates the Stakeholders Identification Diagram (SID), as it was represented in the OpenDesign Platform [3] [11]. SID helps to identify and map different categories of stakeholders potentially affected or affecting the prospective system. They are organized into five layers; operation (those related to the technical operation of the prospective system), contribution (the actors, those who contribute directly to the system), source (clients and information providers), market (collaborators and competitors), and the community (bystanders, legislators).

\subsubsection{Identify Stakeholder Behavior}

This step is focused to identify the stakeholders' behavior with regard to the target behavior defined in the first step. The Stakeholder Behavior Identification artifact is a matrix intended to identify and group the stakeholders according to their typical behavior towards some intended persuasive system. For instance, Figure 4 shows four quadrants: the avoiders are the people who intent to avoid the behavior aimed by the prospective system, only doing it when necessary. Engaged, are the people who are committed to the new behavior due to some illness, work, etc. Aspirants are the people who wish to do the behavior with voluntary intents. Lovers are the people who 
love doing the behavior because they have high involvement with the target behavior (e.g. activists related to sport as a bodybuilder).

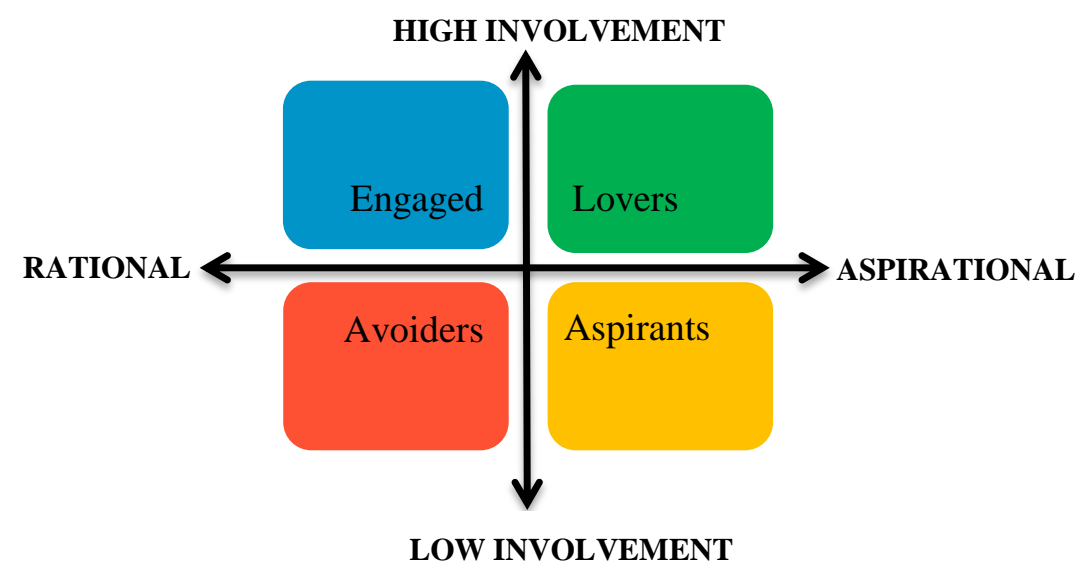

Fig. 4. Stakeholder Behavior Matrix. (adapted from Carvalho [12]).

In the next step, this matrix helps tailoring ideas and solutions for the problems identified for each group of people.

\subsubsection{Find Problems, Propose Ideas and Solutions aided by Persuasive Principles}

This step is supported by the Persuasion Frame (PF) artifact, which provides comprehensive analyzes of the issues that stakeholders might have using the prospective system and helps raising ideas for facing them. Through a collaborative analysis by people working in the OpenDesign Platform [1] [11], this artifact helps to reveal the problems and solutions groups of stakeholders have in a social environment. A list of issues identified and related to the identified interested part that have to be solved are discussed under different points of view, and ideas and solutions to them are raised collaboratively. In this stage of the process, to discuss and propose solutions, persuasive principles are listed, and features are suggested to conceive ideas and project design solutions for the problems discussed. It means solutions or ideas will be associated to persuasive principles. Persuasive principles are described in the next section.

\subsubsection{Persuasive Principles}

Principles are defined as laws that should be followed with certain purposes [16]. In order to design persuasive information systems, in this work persuasive principles are considered as a ground to ideate systems with persuasive aspects.

Principles proposed in this work come from a synthesis of persuasive principles worked in a previous research [4]. Encompassing different aspects of pervasive applications, we identified seven principles: enactive quality, social influence, hedonic 
quality, simplicity, usability, ethical, and accessibility. These principles should be reflected in the system as features and functionalities. Table 1 shows the persuasive principles and features that the systems could adopt to reflect persuasive aspects.

Table 1. Persuasive principles for persuasive design [4].

\begin{tabular}{l}
\hline Enactive Quality \\
\hline Immersion \\
Proactivity \\
Autonomy \\
Sensory motiva- \\
tion
\end{tabular}

\begin{tabular}{|c|c|c|}
\hline Social Influence & Hedonic Quality & Simplicity \\
\hline Social Learning & Emotional motivation & Reduction \\
\hline Social facilitation & Aesthetics & Tunneling \\
\hline Cooperation & Fun & Personalization \\
\hline Competition & & Self-monitoring \\
\hline Recognition & & Simulation \\
\hline Rewards & & Rehearsal \\
\hline Social role & & Similarity \\
\hline Group Tailoring & & \\
\hline Social Comparison & & \\
\hline Normative Influence & & \\
\hline Praise & & \\
\hline Liking & & \\
\hline
\end{tabular}

\begin{tabular}{l}
\hline Usability \\
\hline Suggestion \\
Flexibility \\
User control and freedom
\end{tabular}

\begin{tabular}{lll}
\hline Ethical & & Accessibility \\
\hline Expertise & & Perceptible information \\
Benevolence & Inclusiveness \\
Responsibility & \\
Privacy & \\
Trustworthiness & \\
Authorship Transparency & \\
Third party endorsements & \\
Verifiability & \\
Transparency & \\
\hline
\end{tabular}

Enactive Quality: the system should improve the human behavior through a continuous, ubiquitous, and intelligent accompaniment or as a perceptual and cognitive apparatus extension [19]. This principle supports autonomy, proactivity, immersion, and sensory motivations as system features and capabilities. For example: Tesla cars with autopilot features and full self-driving capabilities. It will made easy travel in car and will prevent transit accidents.

Social Influence: people who love making a behavior can influence other people to make this behavior. For example: people can influence by contributing with information for other people to achieve the desired behavior.

Hedonic Quality: fun, humor or emotional experiences can be factors to influence people in order to reach a behavior. For example: Zombies Run is a video game application to encourage run with the fear. Person simulates a survivor, and emotionally increases the capability to run with the fear for not been caught by zombies.

Simplicity: aims to help humans by simplifying task, to reduce time, steps, and effort in order to make a target behavior. Computer systems can do us the favor of simplify- 
ing tasks in order to achieve the desired behavior [5]. For example: an application that shows the user information about the quantity of calories consumed by day; it can help him/her to monitor and control his/her behavior to consume less calories.

Usability: addresses persuasion through the easy way to achieve a desired behavior through the technologies. Example: an application for healthier eating habits suggests children to eats fruits instead of candies at snack time.

Ethical principles: Persuasion in design brings a great responsibility, due to the issues of potential manipulation arising, i.e. persuasion could be for good as well as for bad intentions [18] [1]. As a consequence, it is vital the importance to include the ethical principle in the process of design, as well as related system features. Great examples are sustainable applications.

Accessibility: this principle is strongly related to universal design. This mean the system should be designed also including people with disabilities [17]. Example: System information to save electricity. Design focused in women, men, children, old people, etc.

The aforementioned persuasive principles were analyzed in a previous work ("Extending Persuasive System Design Frameworks") [4], experimented and validated through a case study ("Motivation, Persuasion and Healthy Eating: a Case Study on a Socially-Aware Persuasive System Design") [22]. Section 3 shows a brief illustration of the results.

\subsubsection{Define Requirements}

After discussing the stakeholders' problems, with the persuasion frame and based on the persuasive principles, requirements are specified drawing on ideas and solutions proposed at the persuasion frame step. These ideas and solutions are inspirations for requirements that will be used to design system prototypes. This step considers functional and non-functional requirements defined collaboratively by the system designer and the participants.

\subsubsection{Design Prototypes}

As a preliminary design, prototypes are built as a model of the persuasive system. Prototypes help the stakeholders (co-designers) to understand the initial set of ideas and solutions proposed at the Persuasion Frame (PF) artifact. System designers are the responsible for building prototypes. Figure 5 shows some screenshots of the prototype being considered in the case study. 


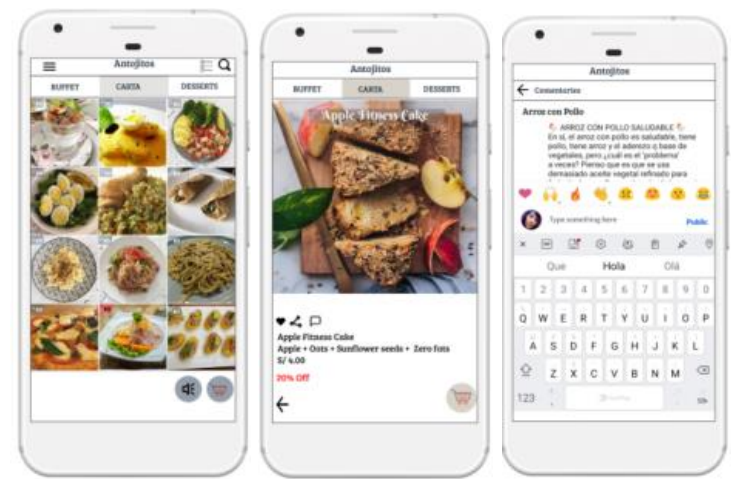

Fig. 5. Some screenshots of prototypes of an application to persuade users to eat healthy food.

The prototypes in Figure 5 show some persuasive features. For instance, the social influence principle, to influence people through other people that consume healthy food (a more detailed explanation is described in a case study, which explains each phase of the system design process and the involved principles [22].

\section{A Brief Illustration of the Framework in a Case Study}

The proposed framework was experimented in a case study to design an information system to motivate and persuade people to eat healthy food in a restaurant. People interested in eating healthy food, restaurant's clients, system designers, systems developers and the restaurant's owner participated as stakeholders in the process to codesign the persuasive system. The five steps proposed in this framework were executed as a process to design the socially aware persuasive information system. Stakeholders participated collaboratively at the design process in each step. After the design, the information system that includes persuasive features in the digital system, as well as its considerations at the social and physical ambience surrounding the person was developed. The system's intention is to generate comfort, with sensorial and emotional experiences to motivate and influence people to eat healthy food. The system designed includes mobile and pervasive applications. Findings of the study surprised us because after experimenting a pilot version of it with a stakeholder that did not have the habit of eating healthy food, at the end of the experiment, the stakeholder asked recipes with vegetables for drinks. Figure 6 shows the experiments being conducted. 


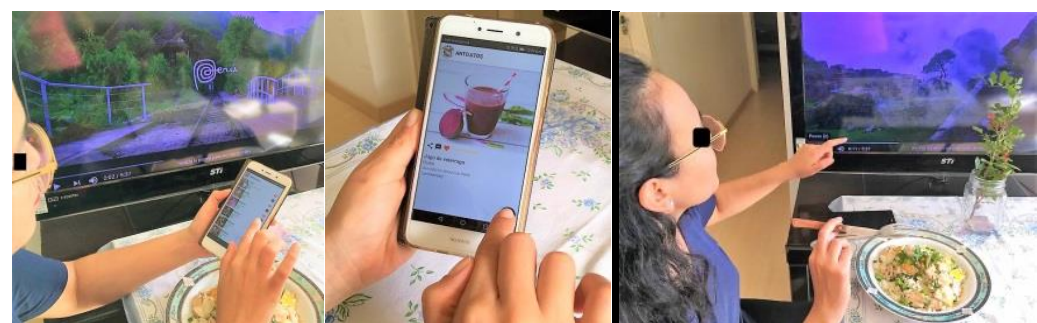

Fig. 6. Case study to persuade people to eat healthy food (Project Management Professional Peru, 2019).

Espinoza and Baranauskas [22] make a deeply explanation of the experimentation with a case study to motivate and persuade people to eat healthy food using this proposed framework. Furthermore, this proposed Framework was evaluated with a research group; as a result the framework was made available online in https://persuasive.opendesign.ml [23].

\section{Conclusion}

The socially aware persuasive system design is a framework that provides artifacts, steps, and tools, to achieve a balance of interests among the interested parties in a prospective system towards the design of information systems with persuasive features. This proposed framework includes the design systems process as a guide to design persuasive systems and its artefacts, based on seven principles. With this research we want to contribute with a formal and practical support to the design of systems with persuasive features.

To analyze the viability of the proposed framework, a case study to motivate and persuade people to eat healthy food is being conducted, although preliminary results have already shown signs of its effectiveness. The system designed in the case study includes mobile and pervasive applications that interact with the person at the social and physical ambiences. The detailed analysis of the case study is going on and will be reported in further work [22].

Ongoing work also involves integrating results of this study in the OpenDesign platform (URL https://persuasive.opendesign.ml), to make available the proposed framework within the practical context of developing information systems, including the pervasive intended ones [23].

\section{$5 \quad$ Acknowledgement}

This research is sponsored by the Council for Scientific and Technological Development (CNPq \#3972865818461011) and Fapesp (2015/24300-9). Also supported by the Computing Institute (IC) from Campinas University (UNICAMP), by the INTERHAD research laboratory group in the Institute of Campinas in Brazil, the Project Management Professional Peru (PMPPeru) and Antojito Restaurant (Peru). 


\section{References}

1. Pereira R., Buchdid S. B., Baranauskas, M. C. C.: Keeping Values in Mind Artifacts for a Value-oriented and Culturally Informed Design. ICEIS $2012-14^{\text {th }}$ International Conference in Enterprise System Information. (2012).

2. Liu, K.: Semiotics in Information Systems Engineering. Cambridge University Press. Cambridge, United Kingdom. (2000).

3. Baranauskas, M. C. C.: OpenDesign : Techniques and Artifacts for Socially Aware Design of Computer System. FAPESP Project \#15/24300-9. (2015).

4. Espinoza, G. E. T., Baranauskas, M. C. C.: Extending Persuasive System Design Frameworks: An Exploratory Study. In International Conference on Information Technology \& Systems (pp. 35-45). Springer, Cham. (2019).

5. Fogg, B.: Persuasive computers: Perspectives and research directions. Proceedings of the SIGCHI Conference on Human Factors in Computing Systems, 225-232. (1998).

6. Oinas-Kukkonen, H., Harjumaa, M.: Persuasive systems design: Key issues, process model, and system features. 24. Communications of the Association for Information Systems, 24(1), Article 28. (2009).

7. Mustaquim, M., Nyström, T.: Designing Persuasive Systems for Sustainability - A Cognitive Dissonance Model. In: Proceedings of the 22nd European Conference on Information Systems (ECIS) 2014 AIS, 9-11. (2014).

8. Stibe, A., Cugelman, B.: Persuasive Backfiring: When Behavior Change Interventions Trigger Unintended Negative Outcomes. In International Conference on Persuasive 2016, 65-77. Springer, Cham. (2016).

9. Murillo, M. M. F., Vazquez, B. M., Cota, C. X. N., Nieto H. J. I.: A Framework for Design and Development of Persuasive Mobile Systems. 2018 International Conference on Electronics, Communications and Computers (CONIELECOMP), 59-66. IEEE. Cholula, Mexico. (2018).

10. Valderlei, S. J., Pereira, R., Bastos, B. S., Duarte, E. F., Baranauskas, M. C. C.: SAwD Socially Aware Design: An Organizational Semiotics-Based CASE Tool to SupportEarly Design Activities. Socially Aware Organisations and Technologies, Impact and Challenges: 17th IFIP WG 8.1 International Conference on Informatics and Semiotics in Organisations, ICISO 2016, proceedings (477), 60-69. Springer, Campinas, Brazil. (2016).

11. Gonçalvez, F. M., Baranauskas, M.C.C., OpenDesign: Analysing Deliberation and Rationale in an Exploratory Case Study submitted paper. Campinas, Brazil. (2019).

12. Carvalho S.: Matriz de envolvimento. Comportamento Online e Gestão da Experiência Omnichannel. Escola de Propaganda e Marketing (ESPM), Brazil. (2019).

13. Baranauskas, M.C.C.: O modelo semioparticipativo de design. In: Baranauskas, M.C.C., et al. (eds.) Codesign de Redes Digitais: Tecnologia a Serviço da Inclusão Social. Penso, Porto Alegre (2013).

14. Valderlei, S. J., Pereira, R., Hayashi, E. C. S., Baranauskas, M. C. C.: Design Practices and the SAwD Tool: Toward the Open Design Concept. Digitalisation, Innovation, and Transformation. 18th IFIP WG 8.1 International Conference on Informatics and Semiotics in Organisations, ICISO 2018. UK. (2018).

15. Liu, K., Li, W.: Organisational Semiotics for Business Informatics. Taylor and Francis, London (2014).

16. Principle. https://en.wikipedia.org/wiki/Principle. Last accessed 2020/04/24

17. Accessibility. https://en.wikipedia.org/wiki/Accessibility. Last accessed 2020/04/26.

18. Kight R., Gram-Hansen S.B.: Do Ethics Matter in Persuasive Technology?. In: OinasKukkonen H., Win K., Karapanos E., Karppinen P., Kyza E. (eds) Persuasive Technology: 
Development of Persuasive and Behavior Change Support Systems. PERSUASIVE 2019. Lecture Notes in Computer Science, vol 11433. Springer, Cham. (2019).

19. Fogg, B.: Creating Persuasive Technologies: An Eight-Step Design Process. In Proceedings of the 4th international conference on persuasive technology, 44-48. ACM. (2009).

20. Kinnear, F.J., Wainwright, E., Bourne, J.E. et al. The development of a theory informed behaviour change intervention to improve adherence to dietary and physical activity treatment guidelines in individuals with familial hypercholesterolaemia (FH). BMC Health Serv Res 20, 27 (2020). https://doi.org/10.1186/s12913-019-4869-4

21. Espinoza, G. E. T., Baranauskas, M. C. C.: Designing Socially-Aware Persuasive Systems: a Proposed Framework. SEMISH - Seminário Integrado de Software e Hardware, XL congresso da Sociedade Brasileira de Computação - CSBC. SBC OpenLib. (2020).

22. Espinoza, G. E. T., Baranauskas, M. C. C.: Motivation, Persuasion and Healthy Eating: a Case Study on a Socially-Aware Persuasive System Design. Paper submitted. (2020).

23. Espinoza, G. E. T., Baranauskas, M. C. C.: A CASE Tool for the Socially-Aware Persuasive System Design toward enhancing the Human Behavior. Paper in process to be submitted. (2020)

24. Samoggia A., Riedel B.: Assessment of nutrition-focused mobile apps' influence on consumers' healthy food behaviour and nutrition knowledge. Food Research International 128 108766. Department of Agricultural and Food Sciences, University of Bologna, Italy. (2019).

25. Flaherty, S. J.: Disrupting routines, facilitating control: exploring a change towards healthier food purchasing behavior using a health app. University College Cork. (2019)

26. Baranauskas, M. Cecília C.: Social awareness in HCI. Interactions (New York, N.Y.), v. 21, p. 66-69. (2014). 